\title{
Localization Analysis of Fluorescent-labeled Aeromonas veronii in the Intestinal Tract of Carp using an in vivo Imaging System
}

\author{
Aki Namba ${ }^{1}$, Nobuhiro Mano ${ }^{2 *}$, Hitomi Hirose $^{2}$ and Teruyuki Nakanishi ${ }^{1}$ \\ ${ }^{1}$ Department of Veterinary Medicine, College of Bioresource Sciences, Nihon University, \\ Kanagawa 252-0880, Japan \\ ${ }^{2}$ Department of Marine Science and Resources, College of Bioresource Sciences, \\ Nihon University, Kanagawa 252-0880, Japan
}

(Received September 27, 2011)

\begin{abstract}
Aeromonas veronii is a Gram-negative opportunistic pathogen in fish. We have previously investigated the intestinal microbiota of carp Cyprinus carpio and revealed that most bacterial isolates with high mucus-binding capability were $A$. veronii. With regard to infection, mucosal adhesion of bacteria is regarded as a prerequisite for colonization and invasion. In the present study, we selected three $A$. veronii strains with different mucus-binding capability and pathogenicity, and compared the colonization capability and localization of these strains in the intestinal tract of carp, using an in vivo imaging system. We showed that only a pathogenic strain was mainly visible around the intestinal bulb and rectum of the intestinal tract at $48 \mathrm{~h}$ after administration. Frozen sections with fluorescence signals revealed that the administered bacteria were localized on the mucosal surfaces and in the lamina propria of the intestinal bulb, whereas they were mainly detected in the remaining intestinal contents in the rectum. These results suggest that the intestinal bulb is the primary region for $A$. veronii to invade and proliferate in healthy carp.
\end{abstract}

Key words: Aeromonas veronii, Cyprinus carpio, CFDA/SE, intestinal tract, in vivo imaging

Aeromonas veronii is a common microorganism in freshwater and estuarine environments, and has been recognized as an opportunistic pathogen of fish (Guzman-Murillo et al., 2000; Vazquez-Juarez et al., 2004). Infection with $A$. veronii causes hemorrhagic septicemia in fish, which is increasingly considered a serious economic problem for the fish-farming industry (Austin and Austin, 1999). In a previous study, we investigated the intestinal microbiota of carp Cyprinus carpio using a culture method, and observed that the most frequently isolated bacterial genus was Aeromonas (384/747 total isolates) (Namba et al., 2007). Detailed characterization of the adhesion capability to intestinal mucus of the isolates by an originally developed in vitro assay system (Namba and Hirose, 2005) revealed that the major population of the high-adhesion group consisted of isolates belonging to $A$. veronii (Namba et al., 2007).

Mucosal adhesion of bacteria is regarded as a prerequisite for colonization and invasion regarding infection. To investigate the pathogenicity of $A$. veronii isolates with adhesive capability, we used carp maintained under no-feeding conditions for 2 days and per-

\footnotetext{
* Corresponding author

E-mail: mano.nobuhiro@nihon-u.ac.jp
}

formed oral administration using bacterial suspensions prepared from selected isolates. The results of these experiments revealed that mortality and symptoms of disease were absent in experimental individuals (Namba et al., 2007), but we confirmed that an isolate (strain CWP11) among eight randomly selected isolates exhibited pathogenicity causing lesions on the intestinal epithelium of carp collected at 7 days after oral administration by subsequent histological analysis (unpublished). In another study, we also showed that strain CWP11 was detected in the intestinal tract of carp for a longer period compared with the other strains without causing lesions (Namba et al., 2008). These results suggest that pathogenetic $A$. veronii has highcolonization capability in the carp intestine, but the researches on the localization and changes of administrated bacteria in the whole intestine were scarce. Here we focused on the in vivo imaging system that can provide imaging of fluorescent and luminescent labels related to cell biology, cancer and infection research. In the present study, we selected three $A$. veronii strains with different mucosal adhesive capability and pathogenicity, and compared the colonization capability and localization of these strains in the intestinal tract of carp using an in vivo imaging system for small animals. 


\section{Materials and Methods}

Bacterial strains

A total of three $A$. veronii strains with low- (strain CCP7) and high-adhesive (strains CWP11 and HPI14) capability to carp intestinal mucus isolated from intestine of healthy carp (Namba et al., 2007) were used in these experiments. Strain CWP11 exhibits pathogenicity causing lesions on the intestinal epithelium of carp, while strain HPI14 does not show this capability. Strain CCP7 exhibits inefficient adhesion to carp intestinal mucus.

\section{Preparation of fluorescent-labeled bacteria}

Each strain was grown aerobically in $50 \mathrm{~mL}$ of tryptic soy broth (TSB; Difco) at $25^{\circ} \mathrm{C}$ with shaking for $12 \mathrm{~h}$. Bacterial labeling was performed when each strain reached cell densities of approximately $10^{9} \mathrm{CFU} / \mathrm{mL}$. The cells were then washed twice by centrifugation with sterile $10 \mathrm{~mm}$ phosphate buffer containing $145 \mathrm{~mm} \mathrm{NaCl}$ (PBS, pH 7.2). Each suspension (approximately $10^{9}$ $\mathrm{CFU} / \mathrm{mL}$ ) was labeled with CFDA/SE (5-[and-6]-carboxyfluorescein diacetate succinimidyl ester; Ex 495, Em $520 \mathrm{~nm}$; Invitrogen) (10 $\mu \mathrm{M}$ final concentration) for $2 \mathrm{~h}$ at $25^{\circ} \mathrm{C}$ in the dark. Following labeling, the cells were washed four times with PBS and resuspended in 1-2 $\mathrm{mL}$ of PBS, sprayed into the commercial dry pellets (Fujiseifun Ltd.) slowly. The viability of the incorporated bacterial cells into pellets was assessed by colony count method with tryptic soy agar (TSA, Difco). Bacterial numbers in pellets inoculated with strains CWP11, HPI14, and CCP7 were $1.4 \times 10^{10}, 0.9 \times 10^{10}$ and $1.8 \times$ $10^{10} \mathrm{CFU} / \mathrm{g}$ of pellet, respectively. We also ascertained the detection limit of fluorescent-labeled bacterial number (CFU) using the in vivo imaging system as described below.

\section{Experimental procedures}

Carp with an average weight of $7.0 \mathrm{~g}$ hatched at the Gunma Prefectural Fisheries Experimental Station were used for this study. Fish were randomly assigned to four $90-\mathrm{L}$ experimental aquariums at a density of 20 fish per aquarium with a flow-through system. The water temperature was held at $25 \pm 1^{\circ} \mathrm{C}$ throughout the experimental period. Fish in the experimental aquariums were not fed for 3 days before oral administration of labeled bacteria, and then dry pellets containing either A. veronii strains CWP11, HPI14 or CCP7 labeled with CFDA/SE were fed once to fish reared in each aquarium at a feeding rate of $4.0 \%$ body weight. In addition, dry pellets without bacteria were administered orally to carp as a control group.

After 6, 12, 24, 48, 72 and $120 \mathrm{~h}$, three fish from each aquarium were anesthetized in $0.02 \%(\mathrm{w} / \mathrm{v})$ solution of ethyl-3-aminobenzoate methanesulfonic acid salt (Sigma-Aldrich), and the fluorescence signals from labeled bacteria were detected by an in vivo imaging system (Maestro $500 \mathrm{FL}$, Kurabow) consisting of a CCD imaging sensor with a Excitation-Emission filter set (Blue filter; Ex 465, Em 515). In the present study, we observed either the organs in dissected fish or removed entire intestinal tract from the intestinal bulb to the anus because the fluorescent signals could not be detected from a whole fish body.

To confirm that the fluorescent signals detected by in vivo imaging system were from the labeled bacteria, the intestinal regions with signals were embedded in OCT compound (Sakura Fine Technical), frozen in dry ice -acetone, and sectioned into a thickness of $10 \mu \mathrm{m}$ with a cryostat (CM1850, Leica). The sections were mounted in non-fluorescence glycerol and observed using a fluorescence microscope (AM-10, Nicon).
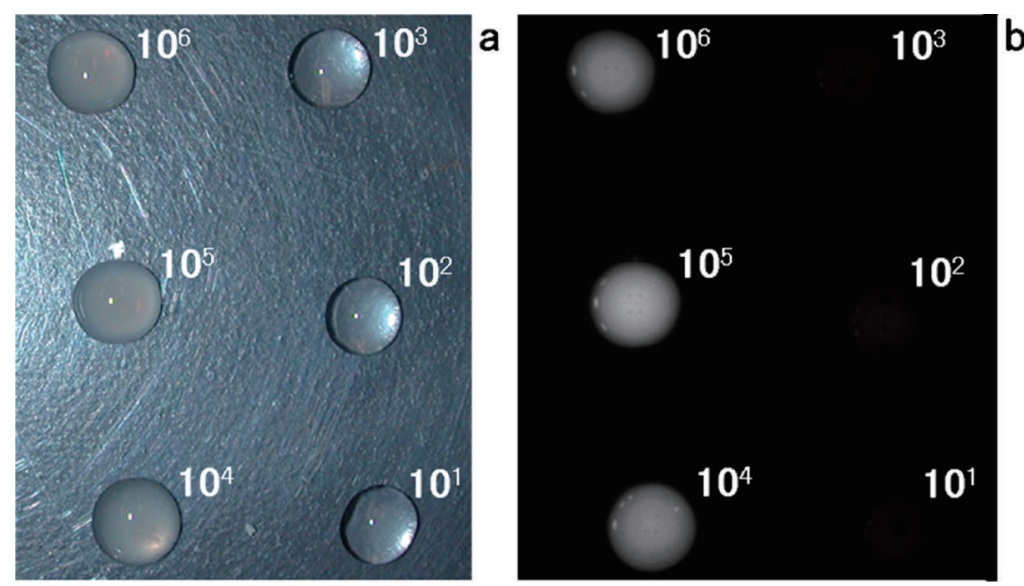

Fig. 1. Example of the relationship between colony forming unit and fluorescent signal of $A$. veronii strain CWP11 labeled with fluorescent marker CFDA/SE. Labeled bacterial suspentions were obsered under brightfield (a) and fluorescence (b) condtions as shown by unmixed images at $515 \mathrm{~nm}$ using an in vivo imaging system (Maestro $500 \mathrm{FL}$, Kurabow). 


\section{Results and Discussion}

The application of in vivo imaging systems is a rapidly growing field, with new technologies and techniques being constantly developed. However, the ability to image fluorescently labeled markers in vivo or ex vivo has generally been limited primarily by the autofluorescence of tissues (Andersson-Engels et al., 1997). In particular, skin and intestinal components have a strong autofluorescence signal when excited in the blue or green wavelengths. In the present study, we therefore obtained spectrally resolved images from 500-720 nm acquisition wavelengths, and performed the localization analysis of fluorescent bacteria by unmixed imaging at $515 \mathrm{~nm}$ which can remove signal degradation caused by autofluorescence.

We first confirmed the fluorescence signal in CFDA/SE-labeled bacterial suspensions (approximately 10 to $10^{6} \mathrm{CFU}$ ) by unmixed imaging as described above. As shown in Fig. 1, it was possible to detect the labeled bacteria on the glass plate, and the detection limit was at least $10^{4} \mathrm{CFU}$.

Figs. 2 and 3 show unmixed images obtained from the dissected body and removed intestines of carp which were orally administered with strains CWP11 or HPI14 after 24 and $48 \mathrm{~h}$, respectively. After $24 \mathrm{~h}$, all strains were visible in the intestine of dissected carp and were mainly localized with the digested diets in the intestinal tract (Fig. 2). On the other hand, strain CWP11 was mainly visible around the intestinal bulb and rectum in removed intestines after $48 \mathrm{~h}$, although strains HPI14 and CCP7 were not detected (Fig. 3). After $72 \mathrm{~h}$ and later, labeled bacteria were not confirmed in all administered fish. Fluorescence signals were also not detected in control fish throughout this experiment. In the frozen sections, the colonies on the mucosal surfaces and invasion into the lamina propria of the labeled bacteria were observed at the intestinal bulb (Fig. 4), while the signals in the rectum were mainly detected in the remaining intestinal contents. These results confirmed the findings of the previous study (Namba et al., 2008) and suggested that an $A$. veronii strain with pathogenicity such as CWP11 has high-colonization capability in the intestinal tract of carp compared with a non-pathogenetic strain. In the previously study (Namba et al., 2008), we compared the composition of membrane proteins between strains CWP11 and HPI14, and detected some adhesion- related membrane proteins that were not from strain HPI14. On the basis of these results, we suggest that the expression pattern of adhesion molecules on the bacterial membrane differs among $A$. veronii strains, and that the colonization capability of bacteria is affected by the differences of these patterns.

It is also suggested that the environments for bacterial colonization of $A$. veronii differ between the intestinal bulb (fore-intestine) and hind-intestine of carp. Although the available histological and biochemical information related to each region of the carp intestine is limited, there are some reports that leucocyte populations

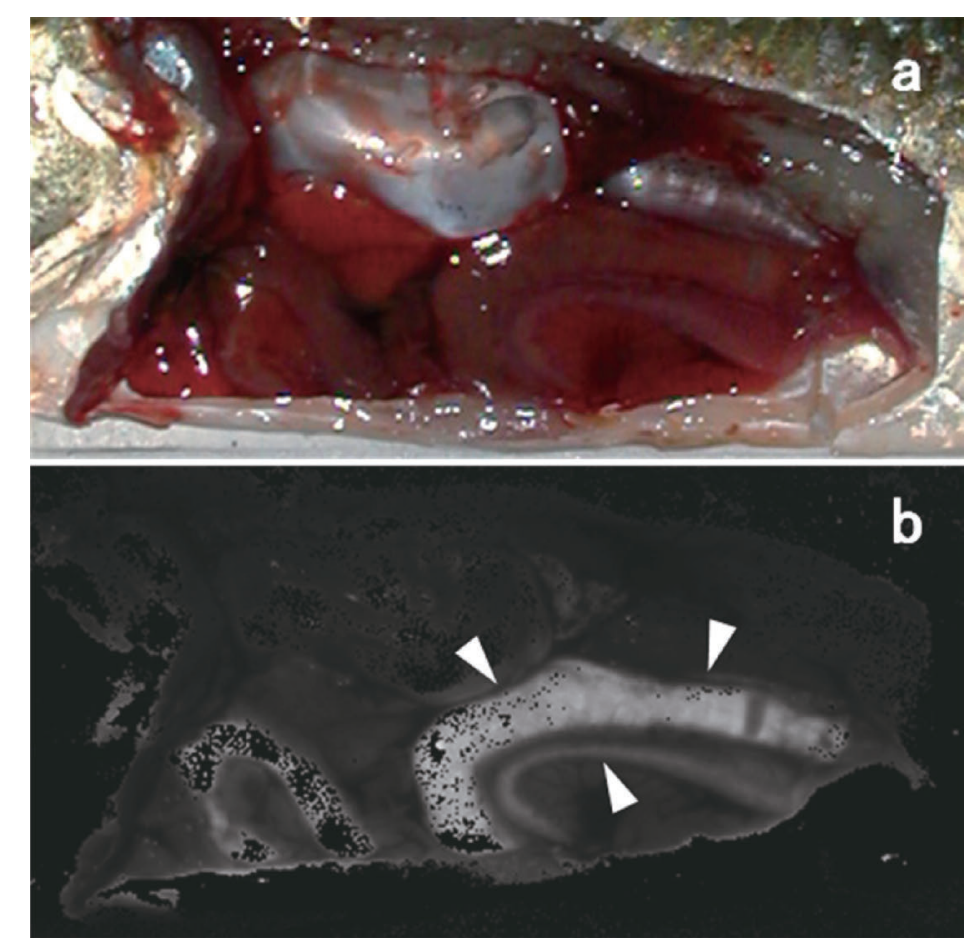

Fig. 2. Organs (a) at $24 \mathrm{~h}$ from carp fed dry pellet containing CFDA/SE-labeled strain CWP11, and an unmixed image (b) at $515 \mathrm{~nm}$. Labeled bacteria (arrow heads; strain CWP-11) were mainly visible in the mid- and hind-intestine. 

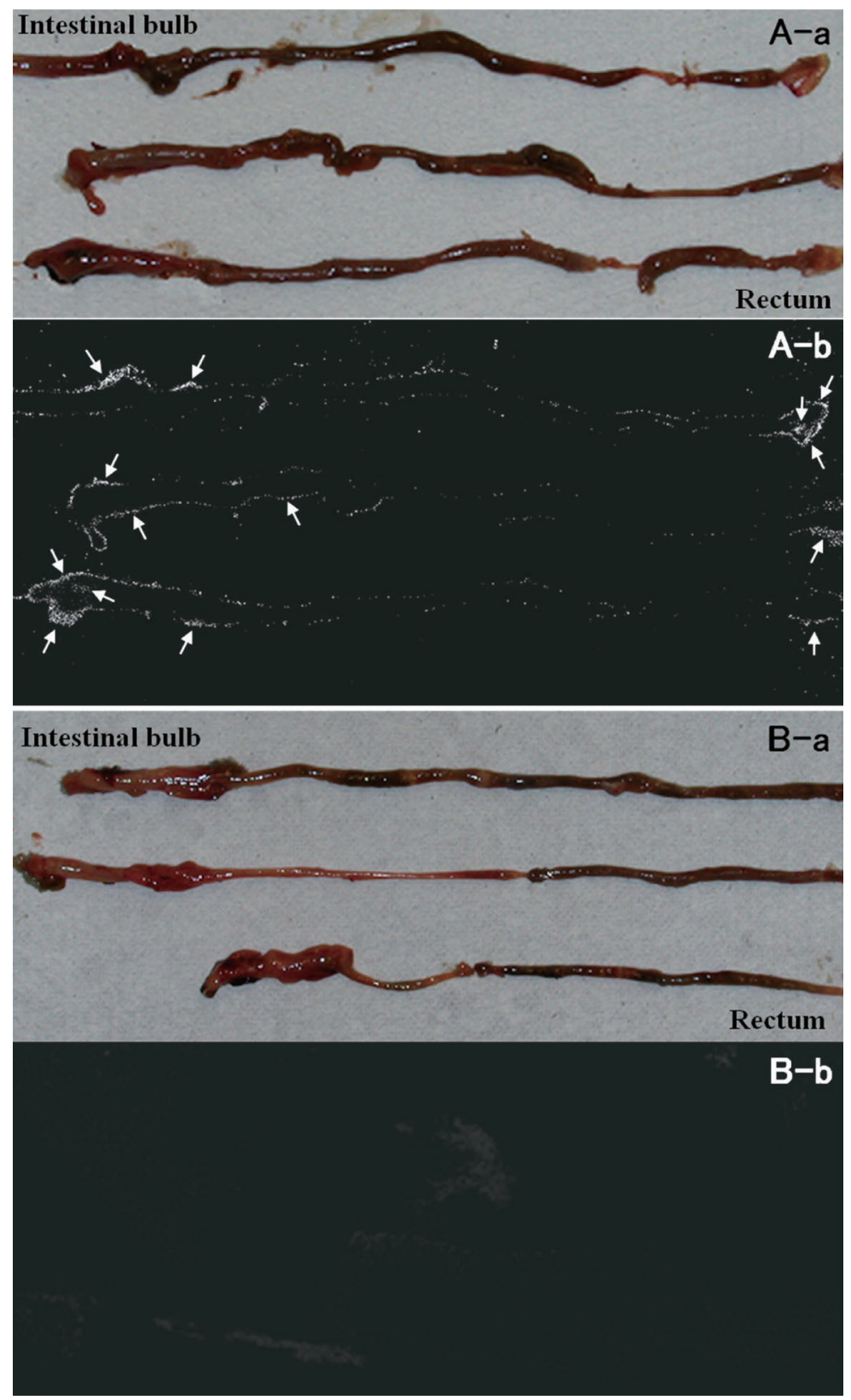

Fig. 3. Intestine (a) removed from carp at $48 \mathrm{~h}$ after oral administration of either strain CWP11 (A) or HIP14 (B), and corresponding unmixed images (b). Labeled strain CWP11 (arrows) was mainly imaged around the intestinal bulb and rectum in removed intestines.

(Rombout et al., 1993) and intestinal microbiota (Namba et al., 2007) differ between the intestinal regions. Our results might indicate that there are differences of mucosal immune-responses in carp and bacterial competition related to colonization of $A$. veronii in each region of the carp intestine, and that the intestinal bulb is the primary region for $A$. veronii to invade and proliferate in healthy carp.

In the present study, we demonstrated that an in vivo imaging system for small animals can more easily estimate intestinal localization and colonization of orally administered bacteria in fish compared with other methods such as histological observation and bacterial isolation. However, the detectable period of this system was shorter than that of the histological observation method using frozen sections in a previous study (Namba et al., 2008). We were also unable to detect fluorescent signals from the whole fish body. A green light (shorter wavelength) fluorescent marker like CFDA/SE used in this study is largely absorbed by tissues, while red light (longer wavelength) penetrates deeper. If we can use an effective fluorescent bacterial marker with a longer wavelength, future analyses are expected to elucidate the infection strategies of fish pathogenic bacteria such as $A$. veronii. 


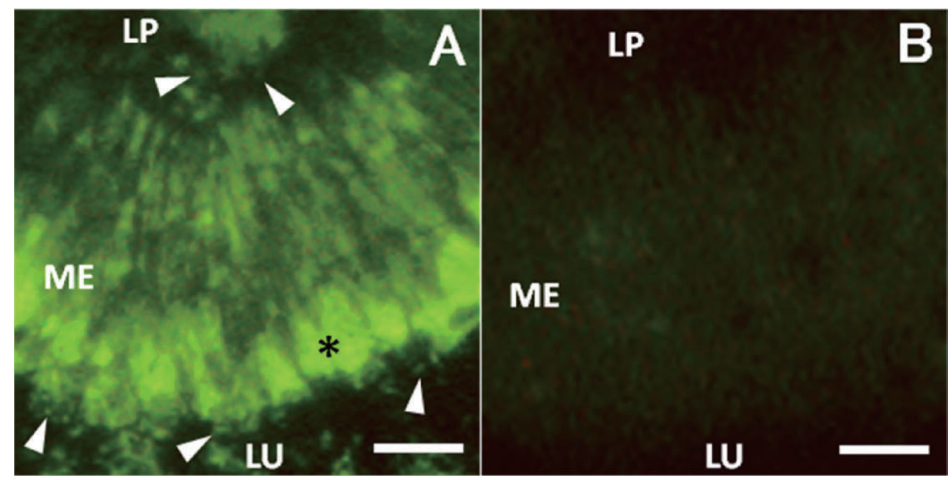

Fig. 4. Fluorescent micrographs showing the presence of $A$. veronii strains CWP11 (A) and HPI 14 (B) in the intestinal bulb prepared at $48 \mathrm{~h}$ after oral administration of each strain. Arrow heads (green signals) indicate the presence of administered bacteria (strain CWP11) on mucosal surfaces and in lamina propria (A), while no signals were detected in tissues administered with strain HPI14 (B). Nonspecific fluorescences (yellow-green signals) containing signals from labeled bacteria were also confirmed in mucosal epithelium of carp administered with strain CWP11 (partially indicated by asterisk). LU: lumen. ME: mucosal epithelium. LP: lamina propria. Bar: $10 \mu \mathrm{m}$.

\section{Acknowledgments}

This study was partially supported by the Academic Frontier Project "Surveillance and Control for Zoonoses" and "High-Tech Research Center" Project for Private Universities: matching fund subsidy from the Ministry of Education, Culture, Sports, Science and Technology of Japan, Global Environment Research Fund of the Ministry of the Environment of Japan (F-062), and Nihon University Research Grants.

\section{References}

Andersson-Engels, S., C. Klinteberg, K. Svanberg and S. Svanberg (1997): In vivo fluorescence imaging for tissue diagnostics. Phys. Med. Biol., 42, 815-824.

Austin, B. and D. Austin (1999): Bacterial Fish Pathogens: Disease in Farmed and Wild Fish, $3^{\text {rd }}$ ed. Springer-Praxis, London, England, $457 \mathrm{p}$.

Guzman-Murillo, M. A., M. L. Merino-Contreras and F. Ascemcio
(2000): Interaction between Aeromonas veronii and epithelial cells of spotted sand bass (Paralabrax maculatofasciatus) in culture. J. Appl. Microbiol., 88, 897-906.

Namba, A. and H. Hirose (2005): A simple microtiter plate assay method for mucosal adhesion of Aeromonas hydrophila and $A$. veronii using a water-soluble tetrazolium salt (WST-1). Aquaculture Sci., 53, 413-417.

Namba, A., N. Mano and H. Hirose (2007): Phylogenetic analysis of intestinal bacteria and their adhesive capability in relation to the intestinal mucus of carp. J. Appl. Microbiol., 102, 1307-1317.

Namba, A., N. Mano, H. Takano, T. Beppu, K. Ueda and H. Hirose (2008): OmpA is an adhesion factor of Aeromonas veronii, an optimistic pathogen that habituates in carp intestinal tract. J. Appl. Microbiol., 105, 1441-1451.

Rombout, J. H. W. M., A. J. Taverne-Thiele and M. I. Villena (1993): The gut-associated lymphoid tissue (GALT) of carp (Cyprinus carpio L.): an immunocytochemical analysis. Dev. Comp. Immunol., 17, 55-66.

Vazquez-Juarez, R. C., M. J. Romero and F. Ascencio (2004): Adhesive properties of a LamB-like outer-membrane protein and its contribution to Aeromonas veronii adhesion. J. Appl. Microbiol., 96, 700-708. 